\title{
The flywheel energy storage for cargo bicycles
}

\author{
Sergey Hoodorozhkov ${ }^{1, *}$ \\ ${ }^{1}$ Peter the Great St. Petersburg Polytechnic University, 29 Politekhnicheskaya street, 195251, Saint \\ Petersburg, Russia
}

\begin{abstract}
This article studies the issues of using in urban conditions a flywheel energy storage for passenger and cargo bicycles (pedicabs) in order to utilization the braking energy of the vehicle for subsequent acceleration by the flywheel. A mechanical stepless self-regulating transmission for the flywheel drive, which allows realize the regenerative braking and acceleration with maximum efficiency, is proposed. The calculation results confirm the effectiveness of proposed technical solution.
\end{abstract}

\section{Introduction}

The urban vehicles - bicycle rickshaws, passenger bicycles, cargo bicycles (pedicabs) have become widespread in densely populated urban areas, fig. 1. The presence of velomobiles with energy recovery system in urban conditions will significantly expand their efficiency. There are two main components are required to implement a recuperative mechanical stepless drive on velomobiles - an energy storage device and a self-regulating mechanical stepless transmission with a wide range of ratios.

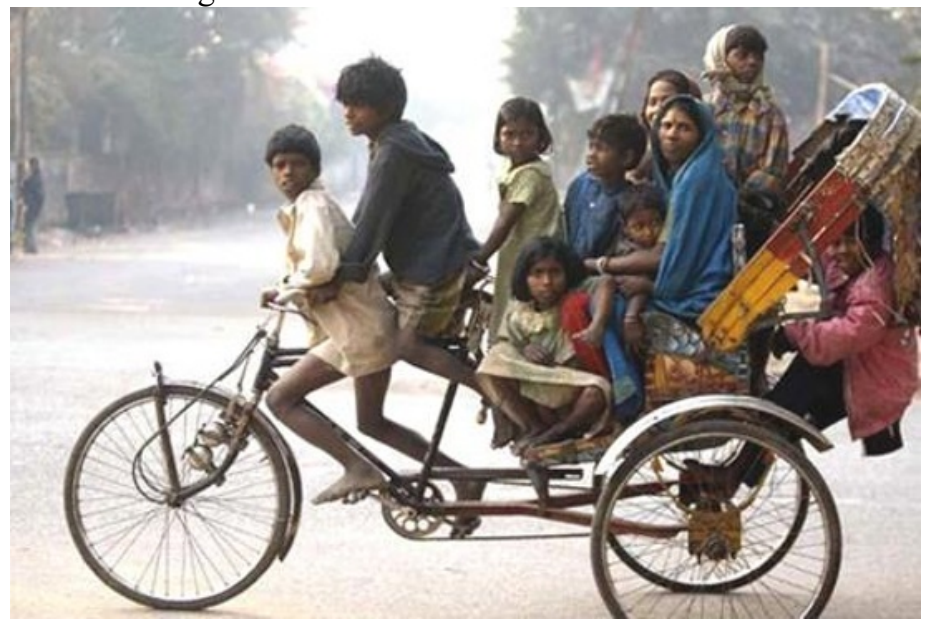

Fig. 1. Pedal-operated pedicab without regenerative system.

\footnotetext{
*Corresponding author: xcu-55@mail.ru
} 
The most suitable for a passenger bicycles, as an energy storage device is a flywheel, since the form of recuperative energy during acceleration and deceleration of flywheel rotation does not change $[1,2]$.

The efficiency of the mechanical drive is as high as possible, and the drive itself is simple and reliable. However, until now, the using of a flywheel energy storage device on a pedicabs was not possible due to the lack of a stepless mechanical drive. The V-belt, disc and ball friction mechanical stepless transmissions can be distinguished among the commercially successful. These transmissions belong to the category of CVT (continuously variable transmission). A distinctive feature of such transmissions is the possibility of continuous regulation of transmission relations in a limited range of output shaft speeds. The main drawback - a small control range $(2 \ldots 4)$ and the need of using an automatic clutch from standing start $[3,4,5,6]$. For these reasons, as well as due to the lack of self-regulation of gear ratio, the inclusion of such transmissions in recuperative system of pedicabs is impossible.

\subsection{Purpose}

Mechanical CVT using of free-wheeling clutches (FWC) are presented by Zero-Max Drives (USA), which supplies the market with a line of commercially successful adjustable variable speed drive [7]. These CVT belong to the category of Infinitely Variable Transmission (IVT). IVT - this is a type of CVT, in which the possibility of transmission ratio change is realized in the infinitely wide range of rotation speeds of the output shaft, including the "stop" mode (fixed output shaft with a rotating input shaft).

For the mechanical drive of the flywheel, it is necessary to use a transmission based on the free - wheeling clutch (FWC), which belongs to the category of stepless drives -Infinitely Variable Transmission (IVT). It has the highest possible efficiency, simplicity and reliability $[8,9,10,11]$.

\section{Methods}

The kinematic diagram of a mechanical self-regulated continuously variable transmission, consisting of drive shaft with a crank 1 , connecting rod 2, rocker arms 3, torsion shaft 4, FWC 5 , output gear 6 , driven shaft 7 and a flywheel 8 is shown on fig.2 $[12,13]$.

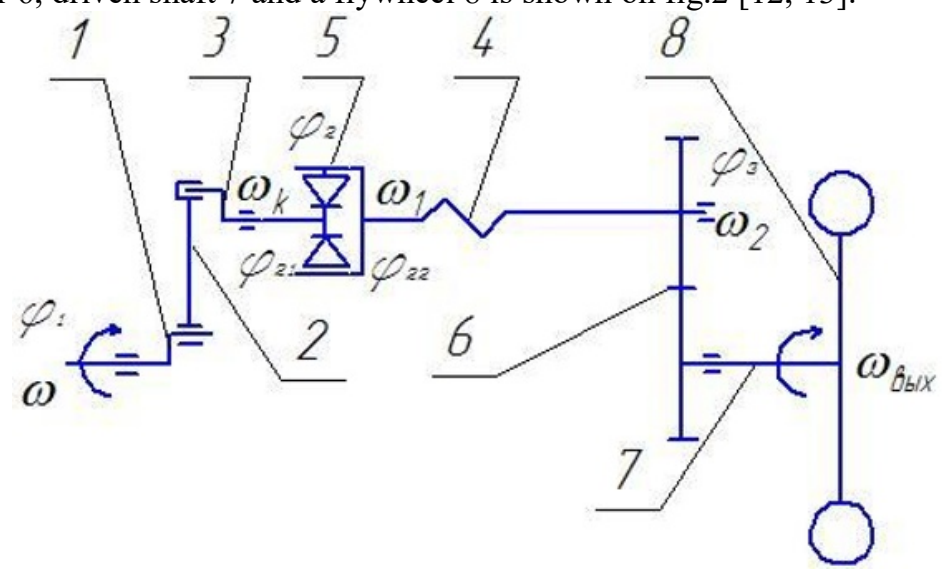

Fig. 2. Kinematic diagram of a mechanical self-regulated infinitely variable transmission.

The external characteristic of transmission in relative coordinates is shown in Fig. 3. The external characteristic has necessary parameters for pedicab: maximum output torque at 
standing start, high torque transformation coefficient, the automaticity of output torque change depending on speed (load) due to the torsion shaft twist and maximum efficiency due by mechanical transformation of torque.

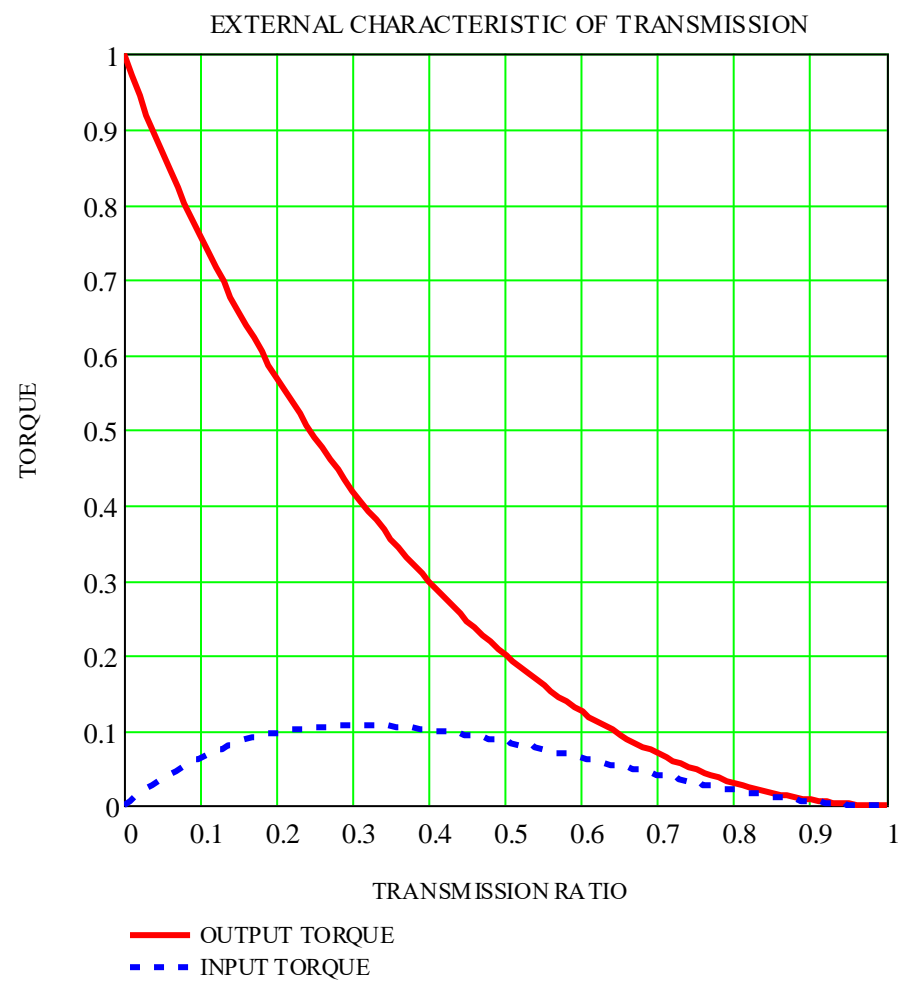

Fig. 3. External characteristic of transmission in relative coordinates.

The kinematic scheme of mechanical system consisting of two identical flywheels connected by two identical but oppositely directed kinematic links is presented on fig. 4 . There are coupling element, four-bars linkage, FWC, torsion shaft and reducer with a constant gear ratio in each circuit. This drive train has the properties of torque converter. 


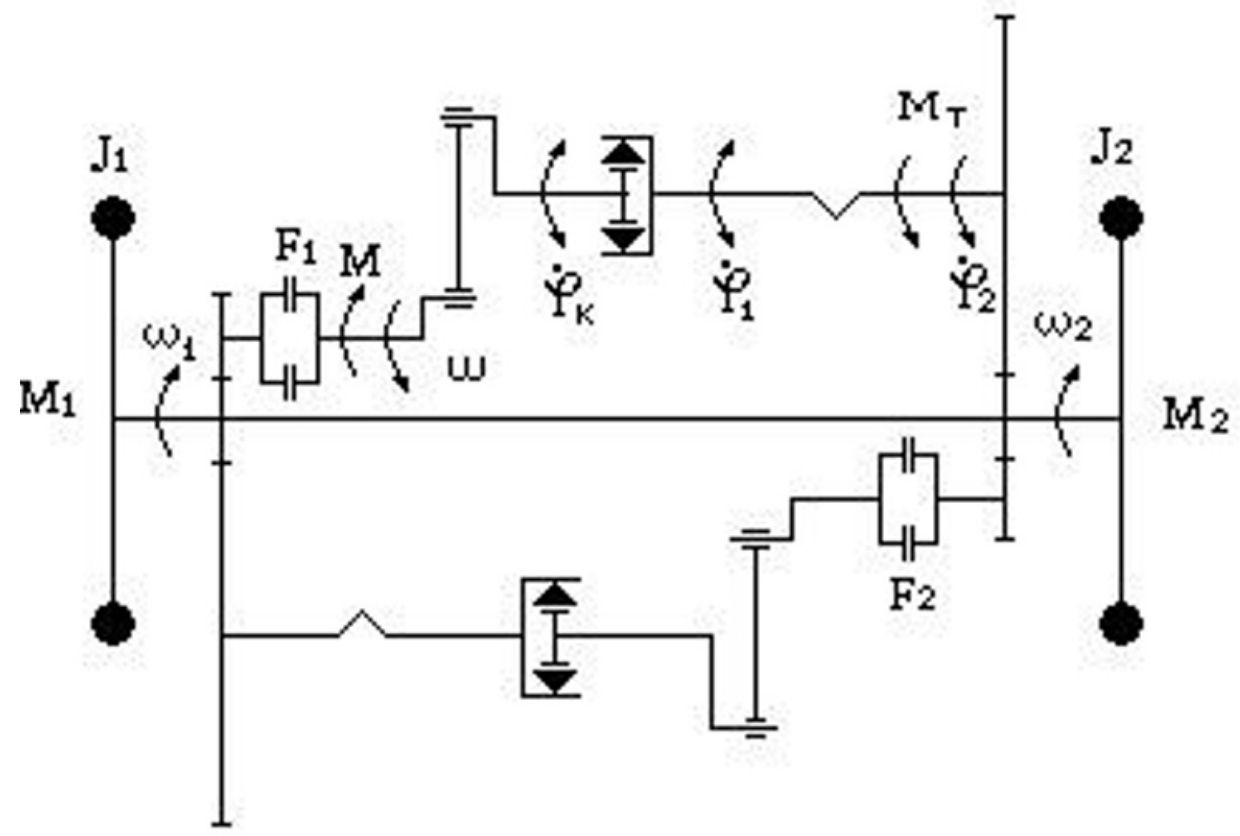

Fig. 4. Mechanical system with two flywheels.

Let consider the energy exchange process between the flywheels when the clutch $F_{1}$ is engaged. In the initial time flywheel J1 has the angular velocity $w_{01}=w_{\max }$, and the flywheel $\mathrm{J}_{2}-w_{02}=w_{\min }$. The flywheel $\mathrm{J}_{1}$ is decelerating and flywheel $\mathrm{J}_{2}$ is accelerating. At the end of deceleration-acceleration cycle should be $w_{1}=w_{\min }$, and $w_{2}=w_{\max }$. The process is exactly the same when the clutch $F_{1}$ is disengaged and the clutch $F_{2}$ engaged, but in the reversal direction. With this in mind can be written:

$$
\left\{\begin{array}{l}
\omega_{\max }=\varphi_{0} \cdot i_{\max } \cdot I_{r_{-} \text {in }} \cdot I_{r_{-} \text {out }} \cdot \omega_{\min } \\
\omega_{\min }=\varphi_{0} \cdot i_{\min } \cdot I_{r_{-} \text {in }} \cdot I_{r_{-} \text {out }} \cdot \omega_{\max } .
\end{array}\right.
$$

Here $i_{\min }$ and $i_{\max }$ are the minimum and maximum values of the transfer ratio $i$, which is an argument of external characteristic (Fig. 3); $I_{r_{-} \text {in }}, I_{r_{-} \text {out }}$ - constant gear ratio of each kinematic chain. On the basis of (1) we have

or else

$$
\frac{\omega_{\max }}{\omega_{\min }}=\varphi_{0} \cdot i_{\max } \cdot I_{r_{-} \text {in }} \cdot I_{r_{-} \text {out }}=\frac{1}{\varphi_{0} \cdot i_{\min } \cdot I_{r_{-} \text {in }} \cdot I_{r_{-} \text {out }}}
$$

$$
\varphi_{0}^{2} \cdot I_{r_{-} \text {in }}^{2} \cdot I_{r_{-} \text {out }}^{2} \cdot i_{\max } \cdot i_{\min }=1
$$

Equation (2) is an expression of the circular transfer ratio of the circuit when both couplings are engaged $\left(F_{1}\right.$ and $\left.F_{2}\right)$. In fact, each time you turn on only one of them. If you set the amplitude of the rocker $\varphi 0$ (constructive parameter) and the value of $i_{\max }$ and $i_{\min }$, then the Equation 2 will allow us to determine the required value $I r_{-}$in, Ir_out and the range of changes the angular velocity of flywheels. Roughly, you can assign $\varphi 0=0.5 \mathrm{rad}$. The values of $i_{\max }$ and $i_{\text {min }}$ should be chosen using an external characteristic (Fig. 2). It is advisable that the values of the moments $M 1$ at $i=i_{\min }$ and $i=i_{\max }$ were approximately equal and not too 
small compared to the maximum value M1, providing the maximum deceleration of the flywheel. You can accept $i_{\min }=0.05 ; i_{\max }=0.7$. Then get

$$
I_{r_{-} \text {in }} \cdot I_{r_{-} \text {out }}=\sqrt{\frac{1}{0.5^{2} \cdot 0.05 \cdot 0.7}}=10.69 \quad D=\frac{\omega_{\max }}{\omega_{\text {min }}}=0.5 \cdot 0.7 \cdot 10.69=3.74
$$

Thus, equation (3) establishes a relationship between the size of the selected range and the values $\varphi 0$ and a constant gear ratio of matching gear Ir_in, Ir_out. This allows to implement a kinematic calculation of pedicab.

The kinematic scheme of the mechanical recuperative transmission of the velomobile is presented on fig. 5. It includs two circuits: the brake recuperative circuit and the accelerating recuperative circuit. The first circuit includes driving wheels 1 and $1 \mathrm{~A}$ on shuft 2 , chainwheel 3, input shaft 4 , sprocket 5, controlled clutch 6 , crank shaft 7 , connecting rod 8 , eccentric shaft 9 , a free-wheeling clutch 10 , torsion shaft 11 , driving sprocket 14 , driven sprocket 16, flywheel 27 . The second recuperative circuit includes a "mirror" drive: flywheel 27 , chain-wheel $3 \mathrm{~A}, 5 \mathrm{~A}$, controllable coupling $6 \mathrm{~A}$, crank shaft $7 \mathrm{a}$, connecting rod $8 \mathrm{~A}$, eccentric shaft 9a, free-wheeling clutch 10A, torsion shaft 11a, chain-wheel 14a, 16A, shaft 2 , drive wheels 1 and $1 \mathrm{~A}$.

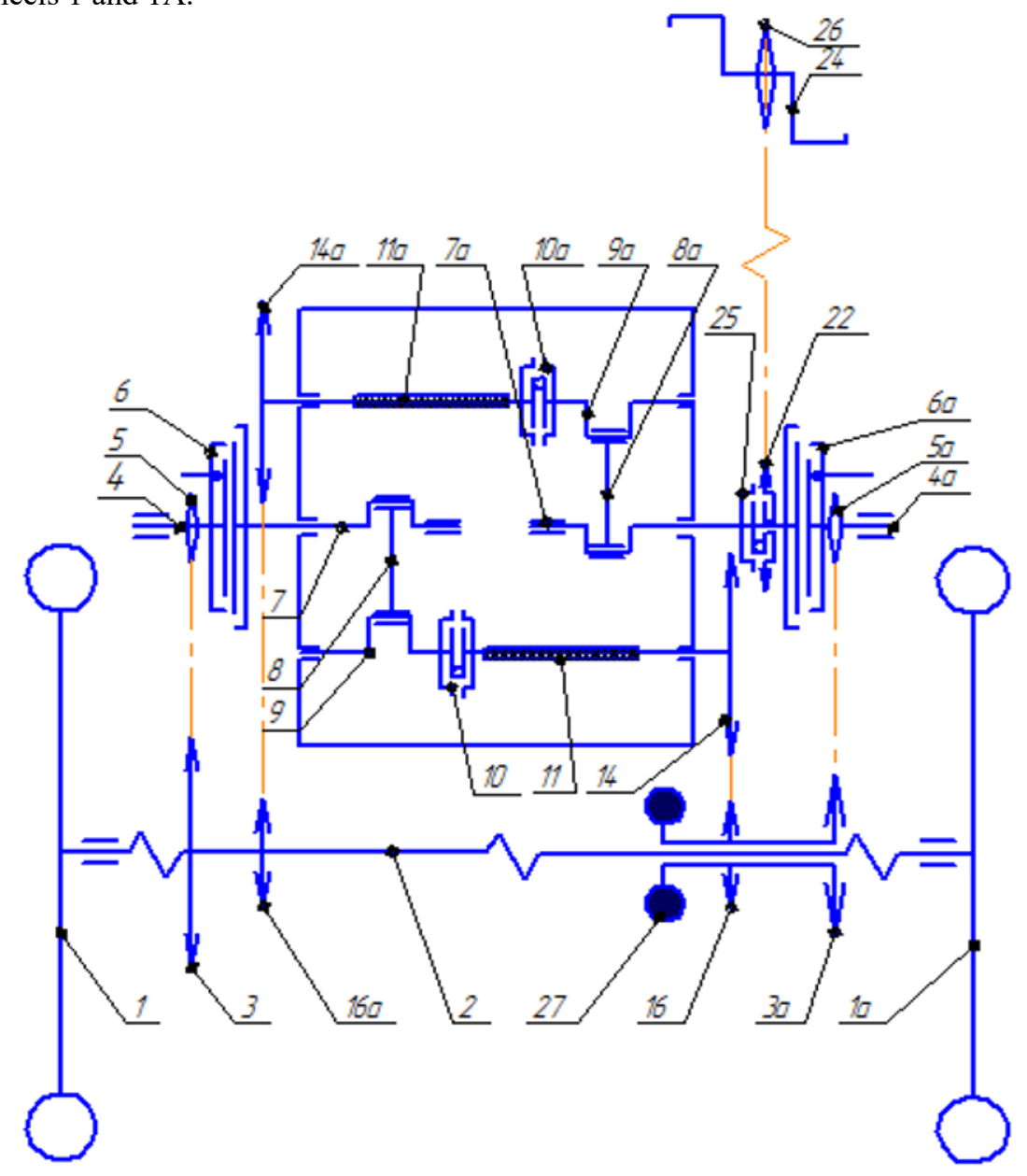


Fig. 5. Kinematic scheme of mechanical recuperative transmission with the flywheel energy storage for pedicab.

\section{Results}

The results of calculation of velomobile's dynamic parameters at recuperative acceleration and braking are presented below and have executed at the following initial data: weight of pedicab $\mathrm{Mp}=260 \mathrm{~kg}$; radius of the drive wheels $\mathrm{Rw}=0.32 \mathrm{~m}$; coefficient of movement resistance $\mathrm{f}=0.012$; maximum speed of pedicab $\mathrm{Vmax}=20 \mathrm{~km} / \mathrm{h}$; mass of flywheel $\mathrm{mf}=10$ $\mathrm{kg}$, maximum speed of flywheel $\mathrm{wf}=2000 \mathrm{rpm}$. Parameters of stepless transmission: amplitude of the eccentric shaft $\varphi_{0}=0.5 \mathrm{rad}$; angular stiffness of torsion shafts $C=350 \mathrm{Nm}$; efficiency $\eta=0.97$.

The two-mass model showing in Fig.6 is used to study the dynamics of recuperative motion of pedicab with stepless flywheel drive $[14,15]$.

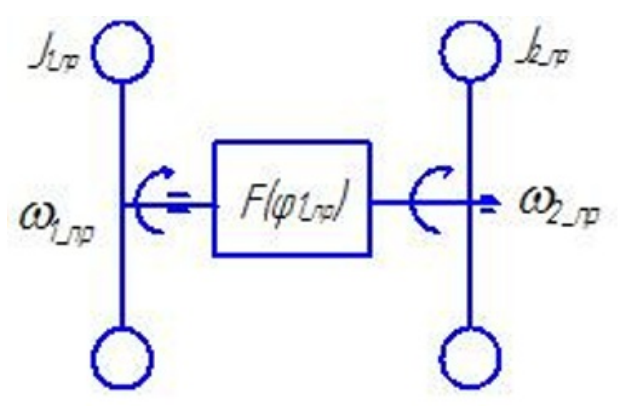

Fig. 6. Two-mass model of pedicab with stepless flywheel drive.

Here $F_{\left(\varphi I_{\perp} p r\right)}$ is a position function (function of generalized coordinates). The first time derivative $\left.F_{(\varphi 1}^{\prime} p r\right)$ - gear ratio.

For the regenerative braking mode by flywheel we obtain the following system of equations:

$$
\left\{\begin{array}{l}
J_{1_{-} n p} \cdot \dot{\omega}_{1_{-} n p}=-M_{c_{-} n p}-M_{T} \cdot F^{\prime}\left(\varphi_{1_{-} n p}\right) \\
J_{2_{-} n p} \cdot \dot{\omega}_{2_{-} n p}=M_{T} .
\end{array}\right.
$$

For the regenerative acceleration mode by flywheel -

$$
\left\{\begin{array}{l}
J_{1_{-} n p} \cdot \dot{\omega}_{1_{-} n p}=-M_{T} \cdot F^{\prime}\left(\varphi_{1_{-} n p}\right) \\
J_{2_{-} n p} \cdot \dot{\omega}_{2_{-} n p}=M_{T}-M_{c_{-} n p} .
\end{array}\right.
$$

Here

$$
\begin{gathered}
M_{c_{-} n p}=\frac{M_{p} \cdot g \cdot f \cdot R_{w}}{i_{1 T} \cdot I_{r_{-} i n}} \\
M_{c_{-} n p}=M_{p} \cdot g \cdot f \cdot R_{w} \cdot i_{2 p} \cdot i_{3}^{\text {- with regenerative braking }} \text { - with regenerative acceleration }
\end{gathered}
$$

Designations for reduction the initial systems $(4,5)$ to the Cauchy form and the numerical solution of the equations are given below:

$$
y_{1}=\varphi_{1}, y_{2}=\dot{\varphi}_{1} \quad y_{3}=\varphi_{2}, \quad y_{4}=\dot{\varphi}_{2}
$$


where $\varphi_{1}, \dot{\varphi}_{1}, \varphi_{2}, \dot{\varphi}_{2}$ - reduced angles of rotation and angular velocities of the input and output shafts of the stepless transmission, respectively.

Taking into account the efficiency of the drive, the initial system of equations of the first order in the mode of regenerative braking will have the form:

$$
\left\{\begin{array}{l}
\frac{d y_{1}}{d t}=y_{2} \\
\frac{d y_{2}}{d t}=-\frac{\left(\frac{M_{c_{-} n p} \cdot I_{r_{-} i n}}{\eta}+\frac{y_{4}}{y_{2}} \cdot \frac{k \cdot C \cdot \varphi_{0} \cdot M_{20}(i)}{I_{r_{-} \text {out }} \cdot \eta}\right)}{J_{1}} \\
\frac{d y_{3}}{d t}=y_{4} ; \\
\frac{d y_{4}}{d t}=\frac{k \cdot C \cdot \varphi_{0} \cdot M_{20}(i) \cdot \eta}{I_{r_{-} \text {out }} \cdot J_{2}}
\end{array}\right.
$$

and, accordingly, in the mode of recuperative acceleration

$$
\left\{\begin{array}{l}
\frac{d y_{1}}{d t}=y_{2} ; \\
\frac{d y_{2}}{d t}=-\frac{\frac{y_{4}}{y_{2}} \cdot \frac{k \cdot C \cdot \varphi_{0} \cdot M_{20}(i)}{I_{r_{-} \text {out }} \cdot \eta}}{J_{1}} \\
\frac{d y_{3}}{d t}=y_{4} ; \\
\frac{d y_{4}}{d t}=\frac{k \cdot C \cdot \varphi_{0} \cdot M_{20}(i) \cdot \eta-\frac{M_{c_{-} n p}}{\eta}}{I_{r_{-} \text {out }} \cdot J_{2}}
\end{array}\right.
$$

The results of computer simulation of the processes of regenerative braking and acceleration of the pedicab, performed using MATLAB, are presented below $[16, \ldots, 20]$. Initially, to assess the correctness of the choice of parameters (symmetry of acceleration and deceleration circuits), we analyze the recuperative processes in the pedicab drive in the socalled "ideal" conditions (in the absence of the resistance's moment $M c \_p r=0$ and the drive efficiency equal to one $\eta=1)$.

In this case, the initial braking conditions of the flywheel will correspond to the speed of the pedicab, equal to $20 \mathrm{~km} / \mathrm{h}$ and the speed of the flywheel 27 (Fig.5), equal to $\mathrm{n}_{\mathrm{w}}=2080 / \mathrm{D}$ $=556 \mathrm{rpm}$, where $\mathrm{D}=3.74$ - the range of velocity changes in the recuperative exchange (3) with a maximum process power $\left(i_{\max }=0.5, i_{\min }=0.75\right)$. 


\section{Discussion}

Graphs of kinetic energies changes of conditional flywheels (reduced masses) during the regenerative braking of the flywheel are shown in Fig. 7. In this case, the kinetic energy of the pedicab, accumulated before braking in $4000 \mathrm{j}$ is reduced at the end of braking to $400 \mathrm{j}$, while the kinetic energy of the flywheel increases from $400 \mathrm{j}$ at the beginning of braking to $4000 \mathrm{j}$ at the end of the process. The process of energy exchange of conditional flywheels takes place within 6 seconds. A similar character has the kinetic energy transformation at recuperative acceleration, but the flywheel and pedicab change places. The symmetry of energy exchange process characterizes the correct choice of the drive design parameters.

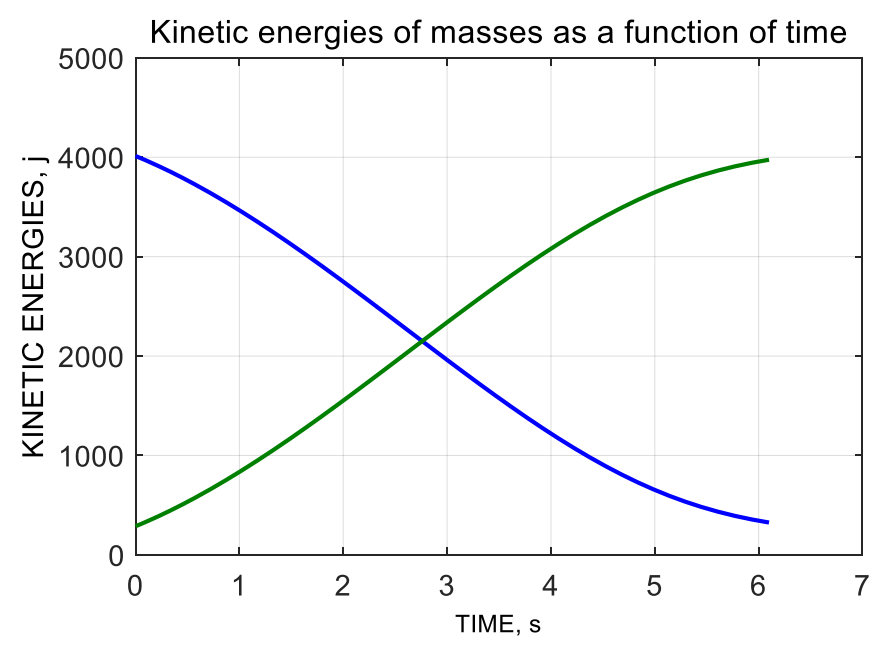

Fig. 7. Parameters of recuperative exchange by kinetic energies of reduced masses in the drive of pedicab under braking in" ideal " conditions.

Characteristics of recuperative processes in the pedicab drive in "real" operating conditions (taking into account the efficiency $\eta \neq 1$ and the moment of resistance Mc $\_p r \neq 0$ ) at braking from $20 \mathrm{~km} / \mathrm{h}$ and "zero" initial speed of the flywheel are discussed below.

The curves of velomobile reducing speed (from 20 to $3 \mathrm{~km} / \mathrm{h}$ ) and the flywheel increasing speed of (from 0 to $1480 \mathrm{rpm}$ ) at the recuperetive braking are shown in figure $8, \mathrm{a}, \mathrm{b}$. The braking distance of pedicab under recuperative braking is shown in Fig. 10A and equals $18 \mathrm{~m}$ in 5.2 seconds.

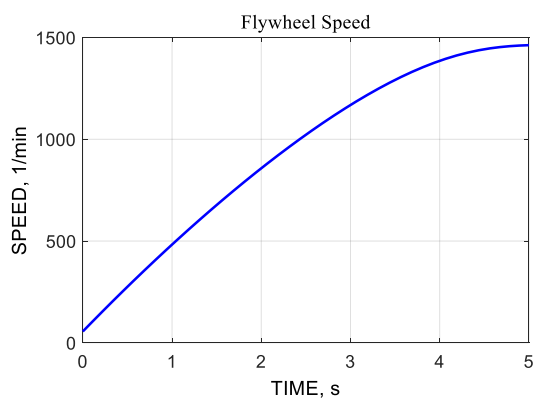

a)

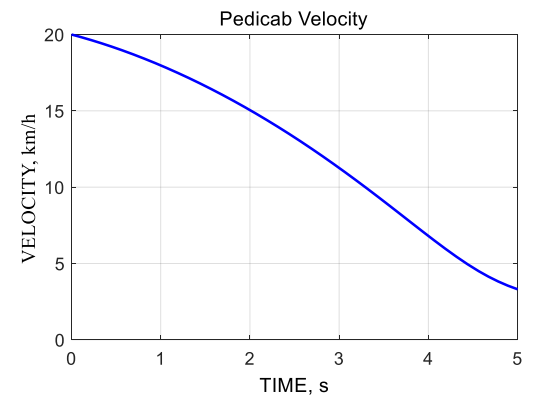

b)

Fig. 8. Graphs of pedicab linear velocity (a) and flywheel speed (b) during recuperative braking. 
The graphics at the process of recuperative acceleration of the pedicab are shown at fig. 9, a, b: speed of the flywheel is reduced from 1450 to $350 \mathrm{rpm}$, and speed of pedicab increases from zero to $12 \mathrm{~km} / \mathrm{h}$. The starting distance is $11 \mathrm{~m}$.

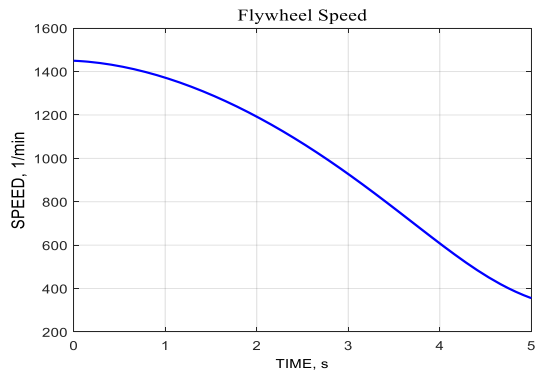

a)

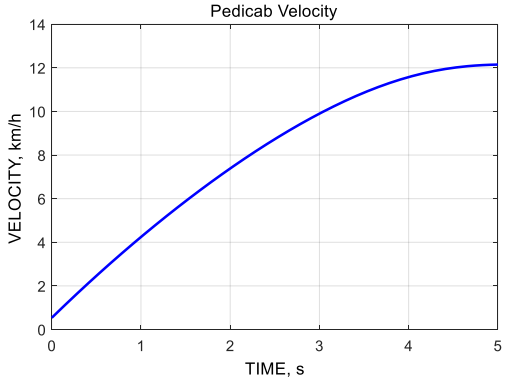

b)

Fig. 9. Graphs of pedicab linear velocity (a) and flywheel speed (b) during recuperative acceleration.

In fact, taking into account the efficiency and expenses on resistance to movement leads to decrease of kinetic energy in comparison with theoretical calculations on $2550 \mathrm{j}$, i.e. on $64 \%$ (approximately, on $32 \%$ in a braking mode and $32 \%$ in a acceleration mode). Accordingly, the speed of pedicab at the beginning of the braking $(20 \mathrm{~km} / \mathrm{h}$, Fig. 8 ,a) and at the end of the acceleration (12 km/h, Fig. 9, a) differs from the theoretical cycle with symmetric energy recovery parameters.

\section{Conclusions}

The flywheel weighing $10 \mathrm{~kg}$, accelerating from 0 to $1500 \mathrm{rpm}$, provides smooth braking of cargo bicycle, weighing $260 \mathrm{~kg}$, from 20 to $3 \mathrm{~km} / \mathrm{h}$ for 5 seconds. The same flywheel due to the accumulated kinetic energy allows to accelerate cargo bicycle of the same mass from 0 to $12 \mathrm{~km} / \mathrm{h}$ in 5 seconds, while reducing speed from 1500 to $350 \mathrm{rpm}$.

The results of calculations is showing that with a single mode of regenerative braking and acceleration, the energy gain will be about $36 \%$. Thus, the effectiveness is very noticeable.

\section{References}

1. Beacon Power Flywheel Energy Storage Systems. www.beaconpower.com.

2. Douglas Cross, Chris Brockbank. Mechanical Hybrid system comprising a flywheel and CVT for Motorsport \& mainstream Automotive applications. 09PFL -0922, Copyright (C) 2008 SAE International. UK, doug.cross@flybridsystems.com. 11 p.

3. Faust, H.; Homm, M.; Bitzer, F.: Optimising the Efficiency of a CVT Clamping System. 7th LuK Symposium (2002).

4. "Reduce energy consumption of belt-driven engine accessories with a $\mathrm{NuVinci}{ }^{\circledR}$ crankshaft mounted Continuously Variable Accessory Drive (CVAD), "http://www.fallbrooktech.com/docs/CVAD_crank.pdf, September 8, (2009)

5. J. Cotrell, Motion Technologies CRADACRD-03-130: Assessing the Potential of a Mechanical Continuously Variable Transmission, NREL/TP-500-36371, September 2004. 
6. S. Akehurst, S.A. Parker, S. Schaaf, Journal of Mechanical Design 129, Nov. , 11701178 (2007)

7. "Zero-Max motion control products," http://www.zeromax.com/products/drives/drivesmain.asp, September 8, 2009.

8. Osw. Friedmann; W. Haas; U. Mair., 7th LUK SIMPOSIUM, 107-116 (2002)

9. Mike Brown, Brent Marquardt. Ker-Train High efficiency drive-by-wire transmission systems., INDIA GROUND VEHICLE SYSTEMS. Engineering and Technology (2017)

10. George Constantinesco:Inertial Tranmission, http://www.rexreseach.com/constran/1 constran.htm.

11. W.T. Lester, Symposium. Power \& Mobility Technical session. August 8-10, - NOVI, Michigan (2017)

12. S. Hoodorozhkov, St. Petersburg Polytechnic university journal of engineering science and technology 3(249), 118-127 (2016)

13. S. Hoodorozhkov, A. Krasilnikov, E. Zakhlebaev. SHS Web of Conferences 44, 00038 (2018)

14. S. Timoshenco D.H. Young, W.Jr. Weaver Vibration problems in engineering. New York: JOHN WILEY \& SONS, Inc., 472, (1974)

15. C.E. Wilson, P. Sadler, Kinematics and Dynamics of Machinery. 3-rd Edition. Pearson Education Limited Edinburgh, 900 (2014)

16. S.I. Hoodorozhkov. Theory and automated control system. (Publising Hous of Polytechnic University, St. Petersburg, 78, (2014)

17. Using Simulink ${ }^{\circledR}$ and Stateflow ${ }^{T M}$ in Automotive Applications. (C) 1998 by The MathWorks, Inc.

18. MATLAB Simulink \&Toolboxes. (2002). http://www.softline.ru.

19. Ivan Graham. MatLAB manual and introductory tutorials. (Publising Hous of University of Bath, 2005), 30 (2005)

20. Simscape User's Guide. (C) COPYRIGHT 2007-2010 by The MathWorks, Inc. (20072010) 